\title{
Assessing the impact of choosing different deformable registration algorithms on cone-beam CT enhancement by histogram matching
}

\author{
Halima Saadia Kidar ${ }^{*}$ and Hacene Azizi
}

\begin{abstract}
Background: The aim of this work is to assess the impact of using different deformable registration (DR) algorithms on the quality of cone-beam $C T$ (CBCT) correction with histogram matching (HM).

Methods and materials: Data sets containing planning $C T(P C T)$ and $C B C T$ images for ten patients with prostate cancer were used. Each $\mathrm{pCT}$ image was registered to its corresponding $\mathrm{CBCT}$ image using one rigid registration algorithm with mutual information similarity metric (RR-MI) and three DR algorithms with normalized correlation coefficient, mutual information and normalized mutual information (DR-NCC, DR-MI and DR-NMI, respectively). Then, the HM was performed between deformed $\mathrm{PCT}$ and $\mathrm{CBCT}$ in order to correct the distribution of the Hounsfield Units (HU) in CBCT images.

Results: The visual assessment showed that the absolute difference between corrected CBCT and deformed pCT was reduced after correction with HM except for soft tissue-air and soft-tissue-bone interfaces due to the improper registration. Furthermore, volumes comparison in terms of average HU error showed that using DR-NCC algorithm with HM yielded the lowest error values of about $55.95 \pm 10.43 \mathrm{HU}$ compared to DR-MI and DR-NMI for which the errors were $58.60 \pm 10.35$ and $56.58 \pm 10.51 \mathrm{HU}$, respectively. Tissue class's comparison by the mean absolute error (MAE) plots confirmed the performance of DR-NCC algorithm to produce corrected CBCT images with lowest values of MAE even in regions where the misalignment is more pronounced. It was also found that the used method had successfully improved the spatial uniformity in the CBCT images by reducing the root mean squared difference (RMSD) between the $\mathrm{pCT}$ and $\mathrm{CBCT}$ in fat and muscle from 57 and $25 \mathrm{HU}$ to $8 \mathrm{HU}$, respectively.

Conclusion: The choice of an accurate DR algorithm before performing the HM leads to an accurate correction of CBCT images. The results suggest that applying DR process based on NCC similarity metric reduces significantly the uncertainties in CBCT images and generates images in good agreement with $\mathrm{pCT}$.
\end{abstract}

Keywords: CBCT images, Deformable registration, Histogram matching, Adaptive radiation therapy

\footnotetext{
* Correspondence: kidar93@yahoo.fr

Department of Physics, Ferhat Abbas Setif University, El Bez Compus, 19000 Setif, Algeria
}

(c) The Author(s). 2018 Open Access This article is distributed under the terms of the Creative Commons Attribution 4.0 International License (http://creativecommons.org/licenses/by/4.0/), which permits unrestricted use, distribution, and reproduction in any medium, provided you give appropriate credit to the original author(s) and the source, provide a link to the Creative Commons license, and indicate if changes were made. The Creative Commons Public Domain Dedication waiver (http://creativecommons.org/publicdomain/zero/1.0/) applies to the data made available in this article, unless otherwise stated. 


\section{Background}

In the past decade, on board cone-beam CT, integrated into linear accelerators was frequently used for image guidance of radiotherapy. It allowed the verification and the correction of patient's setup during the course of treatment in three dimensions with sufficient soft tissue contrast and low patient dose [1-4]. Therefore, it became a powerful tool for improving tumor targeting and reducing dose delivery to normal tissues [5].

Recently, the development of CBCT systems in terms of images acquisition, rapidity and improved image quality has underlined the question of using CBCT images for adaptive radiation therapy (ART). This technique aims to adapt the treatment planning with patient anatomy modification throughout the entire treatment; it is mainly based on three complex and consuming time processes: acquisition of daily $\mathrm{CBCT}$ images for making decision if the re-planning is necessary by comparing them to the CT images, the second process concerns the acquisition of new pCT images and the delineation of volumes of interest to provide a base for the last process which is the dose re-calculation [6]. However, repeated acquisition of CT images for each planning is unjustifiable, due to the accumulated dose. In addition, the preposition of using daily CBCT images directly for dose calculation is limited, owing to their reduced contrast compared to CT images, as shown in Fig. 1, and the large variation of Hounsfield Units caused by the increased amount of scattered radiation [7, 8].

Despite these drawbacks, several studies investigated the feasibility of CBCT images for dose calculation proposing three main "pCT-based" approaches to correct the HUs distribution and minimize as possible the density differences between $\mathrm{CBCT}$ and $\mathrm{pCT}$ to ensure an accurate dose calculation based on CBCT images. The first approach, known as HU mapping, consists of replacing the HUs values in $\mathrm{CBCT}$ by their equivalent points in $\mathrm{pCT}$ after the application of rigid or deformable registration. The accuracy of this approach is strongly dependent on region of body in which it is applied and it is available just for regions where the intra-scan motion and organs deformation are insignificant $[9,10]$. The second approach is the Multilevel Threshold (MLT), it classifies all CBCT pixels with similar HUs into three or four different segments based on pCT. The use of such approach showed a high accuracy especially when combined with DR which minimizes the effect of organs deformation [11-14]. The last approach is the histogram matching (HM) which allows the adjustment of HUs values between CT and CBCT using cumulative histograms. This modification yielded a good agreement between $\mathrm{CT}$ and modified CBCT even for breast and prostate cancer where the intra-scan motion and organs deformation are significant $[13,15]$. Other correction categories can be found in literature such as: "Scatter calibration" and "physics-based" techniques, which aim to directly use CBCT images without recourse to pCT-based strategies using empirical look-up-table (LUT) to calibrate CBCT images [16-18] and scatter measurement or simulation [19-23].

Focusing on pCT-based techniques, the previously cited works showed that the correction accuracy depends on the correspondence between the voxels of $\mathrm{CBCT}$ and pCT images. Therefore, the choice of DR algorithm must be validated.

The present paper aims to evaluate the impact of using different DR algorithms on the accuracy of CBCT enhancement by HM. A dataset containing $\mathrm{CT}$ and $\mathrm{CBCT}$ images for patients with prostate cancer was used to generate corrected $\mathrm{CBCT}$, then, $\mathrm{HUs}$ values were compared for corrected $\mathrm{CBCT}$ and original $\mathrm{CT}$ using different metrics.

The remainder of this paper is organized as follows. In section "Methods and Materials", used data and each

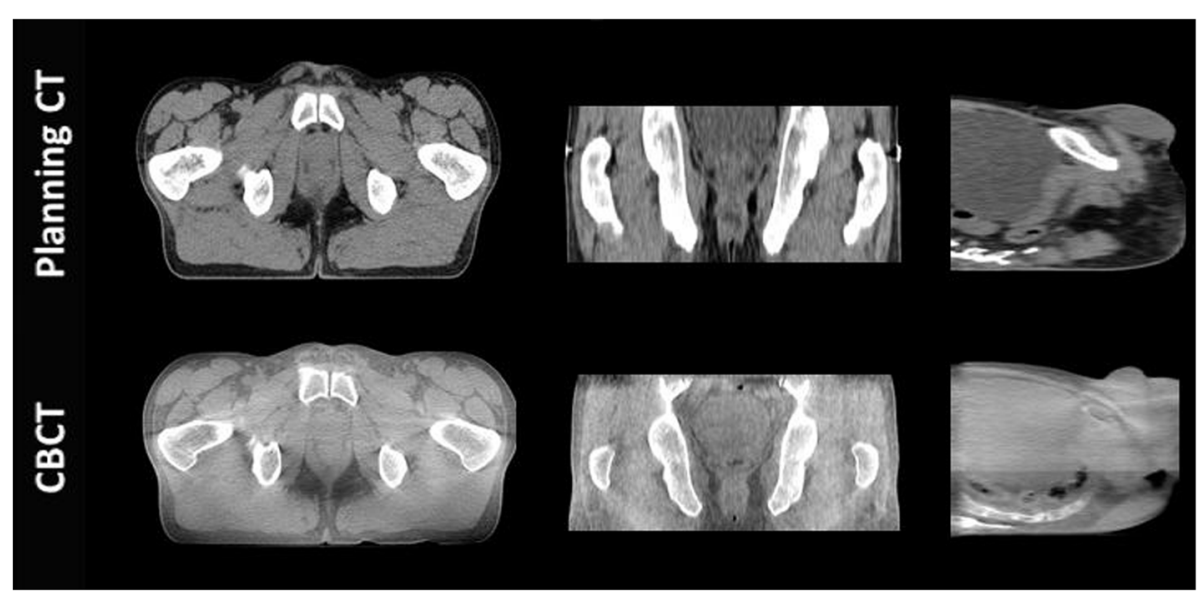

Fig. 1 pCT and CBCT for the same patient (axial, coronal and sagital views) displayed using the same window level 
step to correct $\mathrm{CBCT}$ images are described. Numerical results based on ten prostate cancer patient data sets are presented in section "Results". In section "Discussion", we further discuss the performance and the effect of DR on the correction quality, and finally conclude the paper in section "Conclusion".

\section{Methods and materials}

\section{Data description}

This study was performed on data sets of 10 patients with prostate cancer containing $\mathrm{pCT}$ and $\mathrm{CBCT}$ images obtained by GE CT scan (General Electric Medical Systems) and on-board imager (OBI, Varian Medical Systems) mounted on the gantry of clinical iX21 linear accelerator, respectively. The settings of $\mathrm{pCT}$ and $\mathrm{CBCT}$ acquisition according to pre-defined protocols are recapitulated in Table 1. The slices number differed from a patient to another; it ranged from 123 to 159 slices in the $\mathrm{pCT}$ images and from 50 to 64 slices in the CBCT images giving sufficient information about the anatomical distribution and the motion artifact variations. For all these data $\mathrm{CBCT}$ images were acquired for the first day of treatment to minimize the error of patient's setup under the treatment machine. Since this technique is newly integrated in the clinical practice, the number of patients used in this study is limited.

\section{Images pre-processing}

Initially, collected $\mathrm{CBCT}$ and $\mathrm{pCT}$ contained not only the information describing the patient's body but also the couches of the CT scanner and the linear accelerator. For that reason, all images were pre-processed using the FIJI software [24] to select the region including the patient volume and remove the couches. Furthermore, to eliminate all unnecessary content, a fixed threshold was applied to assign all pixels outside the body surface (below $-700 \mathrm{HU}$ for pCT and bellow $-600 \mathrm{HU}$ for CBCT) to standard CT value for air $(-1000 \mathrm{HU})$ using the 3D Slicer software [25].

\section{Corrected CBCT generation}

In order to assess the impact of DR on the quality of CBCT enhancement, three intensity-based algorithms with different similarity metrics implemented in Elastix [26] were used. The workflow of corrected CBCT generation is described in Fig. 2.

Before starting the DR, the data sets for each patient were aligned using rigid $3 \mathrm{D}$ transformation with mutual information similarity metric (step1 in Fig. 2). Moreover, to minimize the effect of difference in organ deformation between $\mathrm{pCT}$ and $\mathrm{CBCT}$, a multi-resolution B-Spline transformation including 3 levels was performed (step2 in Fig. 2). It is mainly based on the displacement of control points around a control point grid that is put on fixed image, according to the considered similarity metric [26]. The B-Spline interpolator was used to estimate iteratively the deformation field in these points and at each iteration the control points displacement was optimized using the adaptive stochastic gradient descent (ASGD). In this DR process, three similarity metrics were considered: the Normalized Correlation Coefficient (NCC), the Mutual Information (MI), and the Normalized Mutual Information (NMI).

The Normalized Correlation Coefficient (NCC) is given by:

$\operatorname{NCC}\left(I_{F}, I_{M}\right)=\frac{\sum_{x_{i} \in \Omega_{F}}\left(I_{F}\left(x_{i}\right)-\overline{I_{F}}\right)\left(I_{M}\left(T\left(x_{i}\right)\right)-\overline{I_{M}}\right)}{\sqrt{\sum_{x_{i} \in \Omega_{F}}\left(I_{F}\left(x_{i}\right)-\overline{I_{F}}\right)^{2}\left(I_{M}\left(T\left(x_{i}\right)\right)-\overline{I_{M}}\right)^{2}}}$

With $I_{F}$ the fixed image, $I_{M}$ the moving image using a given transformation $T$ and $\left|\Omega_{F}\right|$ is the number of voxels of the fixed image. $\overline{I_{F}}$ and $\overline{I_{M}}$ are the average gray values for the fixed and the moving images respectively.

The Mutual Information (MI) is defined as:

$$
M I\left(I_{F}, I_{M}\right)=H\left(I_{F}\right)+H\left(I_{M}\right)-H\left(I_{F}, I_{M}\right)
$$

Where: $H\left(I_{F}\right)=-\int p_{I_{F}}(a) \log p_{I_{F}}(a) d a$ and $H\left(I_{M}\right)$ $=-\int p_{I_{M}}(b) \log p_{I_{M}}(b) d b$.

With: $\mathrm{H}\left(\mathrm{I}_{\mathrm{F}}\right)$ and $\mathrm{H}\left(\mathrm{I}_{\mathrm{M}}\right)$ the entropies of $\mathrm{I}_{\mathrm{F}}$ and $I_{M}$ respectively. $p_{I_{F}}(a)$ and $p_{I_{M}}(b)$ are the pixel's probabilities with values $a$ and $b$ in $I_{F}$ and $I_{M}$ respectively. $H\left(I_{F} I_{M}\right)$ is the joint entropy of $I_{F}$ and $I_{M}$.

The NMI is given by:

$$
\begin{aligned}
\operatorname{NMI}\left(I_{F}, I_{M}\right) & =1+\frac{M I\left(I_{F}, I_{M}\right)}{H\left(I_{F}, I_{M}\right)} \\
& =\frac{H\left(I_{F}\right)+H\left(I_{M}\right)}{H\left(I_{F}, I_{M}\right)}
\end{aligned}
$$

After DR, the 3D slicer software [25] was used to match the histograms of the CBCT images against the corresponding deformed pCT (step3 in Fig. 2). This processing method aims to adjust the $\mathrm{HU}$ values between $\mathrm{pCT}$ and $\mathrm{CBCT}$ images using their cumulative

Table 1 Acquisition settings

\begin{tabular}{llllll}
\hline Protocol & Tube current $(\mathrm{mA})$ & Exposure time $(\mathrm{ms})$ & Tube voltage $(\mathrm{kVp})$ & Axial image size $($ pixels $)$ & Voxel size $\left(\mathrm{mm}{ }^{3}\right)$ \\
\hline $\mathrm{CT}$ & 360 & 500 & 100 & $512 \times 512$ & $0.8496 \times 0.8496 \times 3$ \\
$\mathrm{CBCT}$ & 80 & 8632 & 125 & $512 \times 512$ & $0.8789 \times 0.8789 \times 2.5$ \\
\hline
\end{tabular}




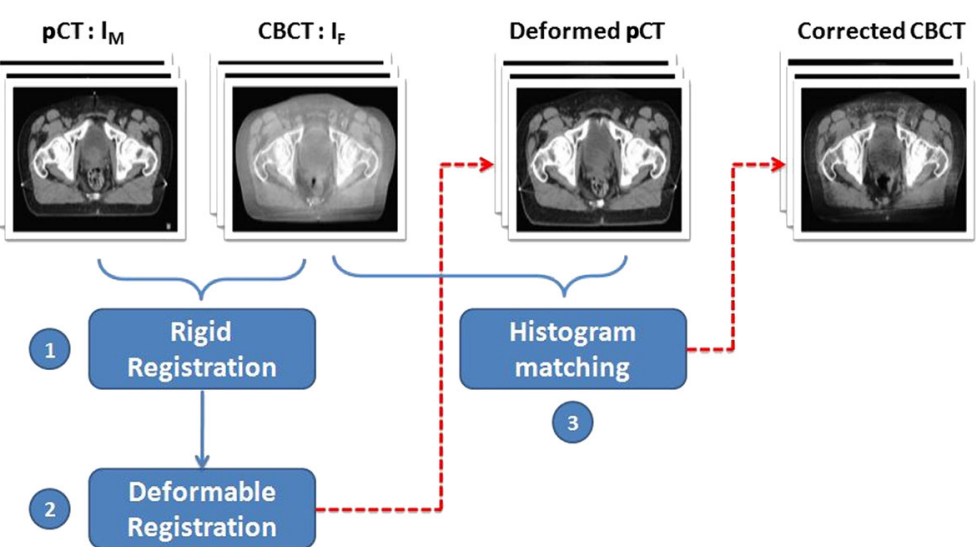

Fig. 2 Workflow of corrected CBCT generation

histograms. Each pixel value in the $\mathrm{CBCT}$ images is replaced by the HU having the same cumulative value in the $\mathrm{pCT}$ images according to the following formula:

$$
\operatorname{CBCT}\left(H_{1}\right)=p C T\left(H_{2}\right)
$$

Where $C B C T\left(H_{1}\right)$ represents the $\mathrm{HU}$ values for CBCT and $p C T\left(H_{2}\right)$ represents the $\mathrm{HU}$ values for $\mathrm{pCT}[13,15]$.

\section{Data analysis}

To evaluate the quality of corrected CBCT, deformed pCT images were considered as a reference for each patient. A visual assessment was performed by the calculation of absolute difference between $\mathrm{pCT}$ and $\mathrm{CBCT}$ images before and after HM to assess the discrepancies between them.

Furthermore, to evaluate quantitatively the agreement between corrected $\mathrm{CBCT}$ and $\mathrm{pCT}$, three methods were used. The first one consists of the average HU error estimation over the entire volume [27] given by:

$$
V_{\text {err }}=\sqrt{\operatorname{mean}\left(\left[H U_{p C T}(x, y, z)-H U_{C B C T}(x, y, z)\right]^{2}\right)}
$$

The second method is the Mean Absolute Error (MAE) plots creation which allows comparing the different tissue classes. It is based on the calculation of the MAE between $\mathrm{pCT}$ and corrected $\mathrm{CBCT}$ in equidistant bins across the HU scale. For this comparison a size of $20 \mathrm{HU}$ was taken for each bin and the formula describing the MAE is given by:

$$
M A E=\frac{1}{N} \sum_{0}^{N}\left|H U_{p C T}-H U_{C B C T}\right|
$$

Where $\mathrm{N}$ is the number of pixels having intensities in [HU-10, HU + 10] in the pCT [28].
The third one is the image quality evaluation in terms of spatial uniformity. For this method, the mean pixel value among five regions of interest (ROIs) having 10 by 10 pixels and positioned in regions of the same soft tissue area is measured [29]. Then, the RMSD between the mean pixel values in the $\mathrm{pCT}$ and the $\mathrm{CBCT}$ images before and after correction are calculated.

\section{Results}

\section{Visual assessment}

Figure 3 shows the absolute difference between deformed $\mathrm{pCT}$ and $\mathrm{CBCT}$ images for one patient before and after HM using three DR algorithms (DR-NCC, DR-MI and DR-NMI). Obtained results for a RR algorithm are also included to confirm the effect of morphologic deformation between $\mathrm{pCT}$ and $\mathrm{CBCT}$ on the quality of correction. The effect of applying $\mathrm{HM}$ is clearly visualized; it reduced the amount of artefacts in CBCT and yielded corrected images in good agreement with deformed $\mathrm{pCT}$. However, high differences in bony regions and soft tissue-air interfaces are present due to the misalignment between $\mathrm{CBCT}$ and $\mathrm{pCT}$.

\section{Volumes comparison}

Results of volumes comparison for each patient in terms of $\mathrm{HU}$ average error between deformed $\mathrm{pCT}$ and $\mathrm{CBCT}$ before and after correction are shown in Table 2. The mean and the standard deviation are also presented.

The largest magnitude of $\mathrm{V}_{\text {err }}$ is observed for unprocessed CBCT images especially when using RR process where the mean HU error value was about $206.47 \pm$ $52.21 \mathrm{HU}$. For the DR process, a significant decrease was obtained with error values ranging from $64.15 \pm$ 9.50 to $68.20 \pm 10.12 \mathrm{HU}$ which confirms the performance of DR algorithms. Whereas, after the correction of CBCT images reduced values of $\mathrm{HU}$ of about $55.95 \pm$ $10.43 \mathrm{HU}, 56.58 \pm 10.51 \mathrm{HU}$ and $58.60 \pm 10.35 \mathrm{HU}$ were obtained for DR-NCC, DR-NMI and DR-MI, 


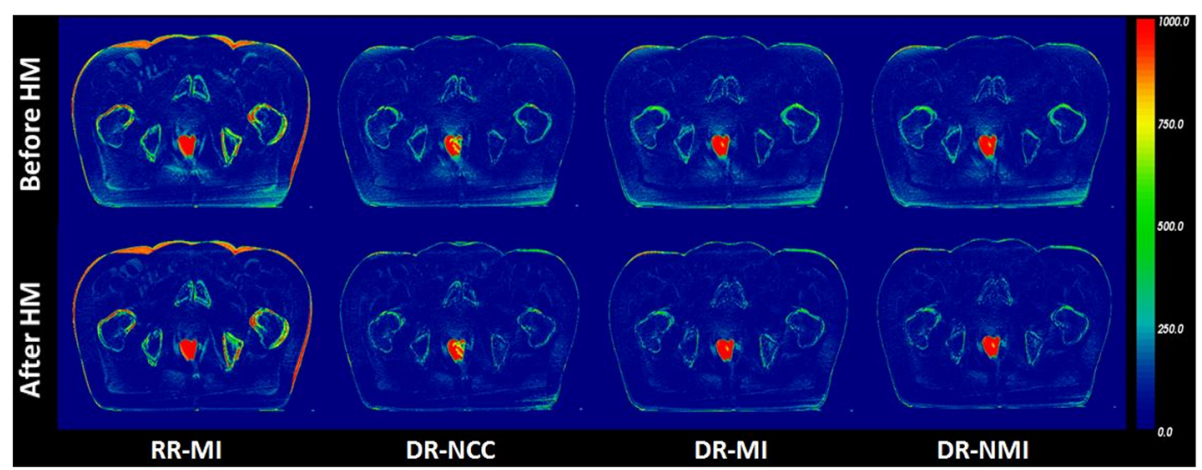

Fig. 3 Absolute difference between deformed $\mathrm{PCT}$ and $\mathrm{CBCT}$ in the first row and corrected $C B C T$ in the second row using one RR and three DR algorithms. Blue colors represent low discrepancies while red colors represent the highest ones

respectively, indicating that the $\mathrm{HM}$ after using DR-NCC yielded corrected CBCT images in good agreement with $\mathrm{pCT}$ images compared to unprocessed CBCT images.

\section{Tissue class's comparison}

Since volumes comparison may not give information about the presence of large errors and their location, the MAE plots for each algorithm over the HU scale are illustrated in Fig. 4. Similarly to [13], HU scale for pCT images was divided according to the tissue type on different classes. All the values lower than $-400 \mathrm{HU}$ was considered as air. The HU values between -400 and 250 HU were associated to soft tissues, while those between 250 and $600 \mathrm{HU}$ presented the soft bone. The remaining values (higher than $600 \mathrm{HU}$ ) were considered as bone.

Figure 4a compares MAE results using RR algorithm before HM with those obtained after HM. It shows obviously that the use of HM with RR increases the uncertainties in CBCT images, due to the misalignment between pCT and CBCT images. However, in (Fig. 4b, c, d) the MAE becomes lower and the combination of DR with HM contributes significantly to reduce the errors after correction, especially for pixels with CT number higher than $200 \mathrm{HU}$. For the values below - $200 \mathrm{HU}$ a mismatch is observed and the MAE values after correction are higher than before. This is due to the low number of pixels in corrected CBCT containing the same $\mathrm{HU}$ values as pCT in the interfaces soft tissue-air, which is in agreement with the visual assessment where high errors were noticeable in those regions owing to the improper registration.

The DR performance comparison is depicted in Fig. 5. Plotting together the MAE values before and after correction against each other (Fig. $5 \mathrm{a}$ and $\mathrm{b}$ respectively) shows that the use of DR based on NCC metric was

Table $2 \mathrm{HU}$ average error values between deformed $\mathrm{PCT}$ and CBCT before and after HM for each patient with the mean and the standard deviation

\begin{tabular}{|c|c|c|c|c|c|c|c|c|}
\hline \multicolumn{9}{|l|}{$\mathrm{V}_{\mathrm{err}}[\mathrm{HU}]$} \\
\hline \multirow{2}{*}{$\begin{array}{l}\text { Patient's } \\
\text { number }\end{array}$} & \multicolumn{2}{|l|}{ RR-MI } & \multicolumn{2}{|l|}{ DR-NCC } & \multicolumn{2}{|l|}{ DR-MI } & \multicolumn{2}{|l|}{ DR-NMI } \\
\hline & Before HM & After HM & Before HM & After HM & Before HM & After HM & Before HM & After HM \\
\hline 1 & 138.50 & 141.33 & 71.03 & 64.70 & 75.94 & 68.07 & 73.53 & 65.67 \\
\hline 2 & 140.00 & 143.01 & 57.89 & 49.10 & 60.77 & 51.76 & 59.90 & 49.87 \\
\hline 3 & 260.62 & 288.10 & 73.58 & 65.17 & 76.33 & 66.71 & 75.09 & 64.84 \\
\hline 4 & 257.67 & 274.16 & 65.76 & 55.10 & 71.23 & 57.05 & 68.51 & 54.03 \\
\hline 5 & 239.04 & 267.54 & 67.48 & 55.73 & 73.34 & 59.89 & 71.44 & 58.02 \\
\hline 6 & 258.85 & 269.82 & 82.09 & 77.88 & 87.37 & 80.24 & 86.24 & 79.04 \\
\hline 7 & 171.96 & 195.80 & 57.74 & 47.26 & 60.12 & 48.18 & 58.90 & 46.26 \\
\hline 8 & 193.20 & 194.09 & 54.89 & 45.74 & 58.68 & 47.75 & 57.23 & 46.03 \\
\hline 9 & 153.75 & 173.61 & 51.82 & 45.90 & 56.13 & 51.47 & 54.33 & 49.09 \\
\hline 10 & 251.15 & 269.41 & 59.29 & 52.92 & 62.16 & 54.96 & 60.62 & 53.01 \\
\hline Mean & 206.47 & 221.68 & 64.15 & 55.95 & 68.20 & 58.60 & 66.57 & 56.58 \\
\hline SD & 52.21 & 57.97 & 9.50 & 10.43 & 10.12 & 10.35 & 10.06 & 10.51 \\
\hline
\end{tabular}



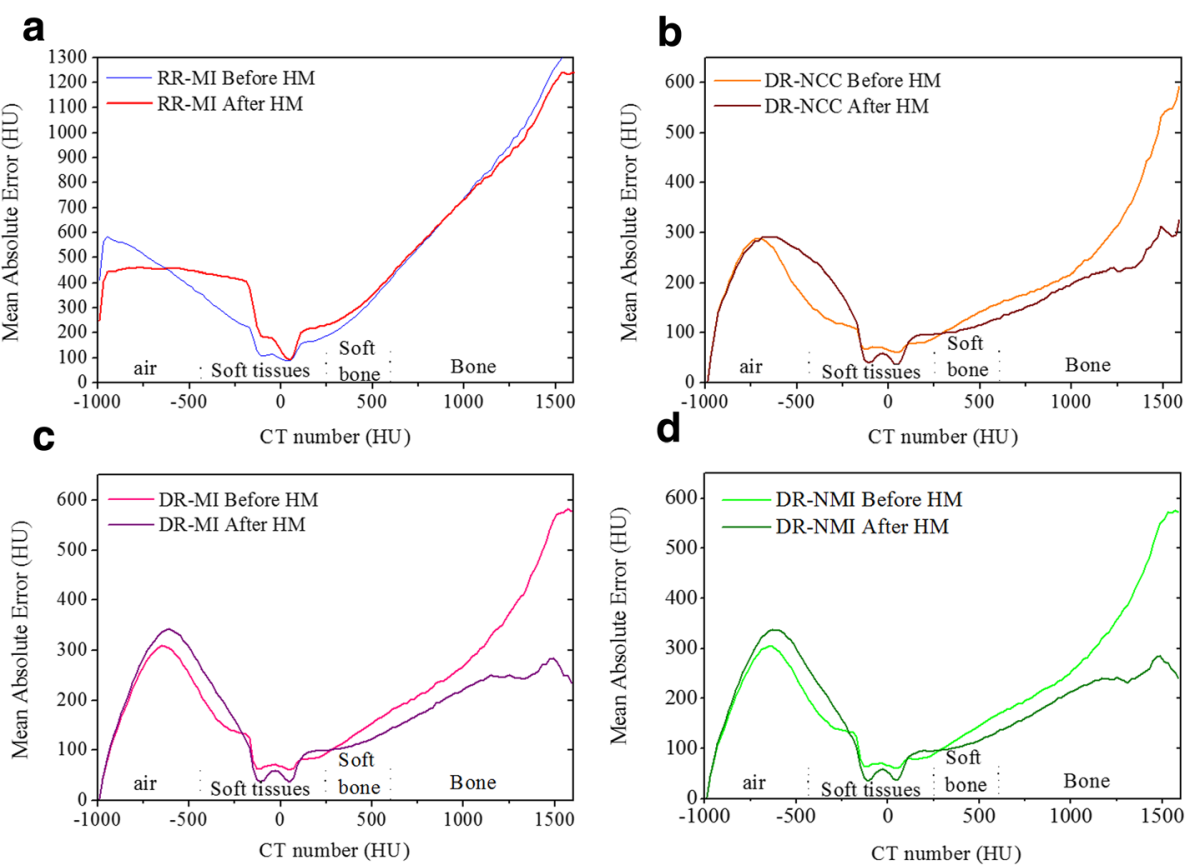

Fig. 4 MAE values of CBCT images before and after correction using a) RR-MI, b) DR-NCC, c) DR-MI and d) DR-NMI

better than MI and NMI especially in soft tissue-air interfaces. Moreover, using the NCC metric in combination with $\mathrm{HM}$ produced more accurate $\mathrm{CBCT}$ images.

Concerning the uniformity of resulted CBCT images, the RMSD of the mean pixel values of ROIs between $\mathrm{CBCT}$, corrected $\mathrm{CBCT}$ and $\mathrm{pCT}$ images are summarized in Table 3. The obtained results showed that the use of HM reduced the RMSD in fat and muscle (soft tissues) from about 57 and $25 \mathrm{HU}$ to $8 \mathrm{HU}$, respectively, indicating that the $\mathrm{CBCT}$ image quality was brought closer to the $\mathrm{pCT}$ image quality through this correction technique.

\section{Discussion}

In this work, the impact of choosing different registration algorithms on the quality of $\mathrm{CBCT}$ correction by
HM was studied. One RR algorithm based on MI similarity metric and three DR algorithms including NCC, MI and NMI similarity metrics were validated.

Several studies investigated the accuracy of dose calculation based on corrected CBCT using HM with DR based on MI $[13,15]$ but our strategy differs from those studies because it aims to initially choose the appropriate DR algorithm, and then generate corrected CBCT images.

All the results confirmed that the performance of DR of each algorithm is strongly dependent on the region in which the transformation was applied. It was shown that all DR algorithms provided a good alignment between anatomical structures in $\mathrm{PCT}$ and $\mathrm{CBCT}$ compared to $R R$ registration but their reduced ability to align some regions as soft tissue-air and soft tissue-bone interfaces
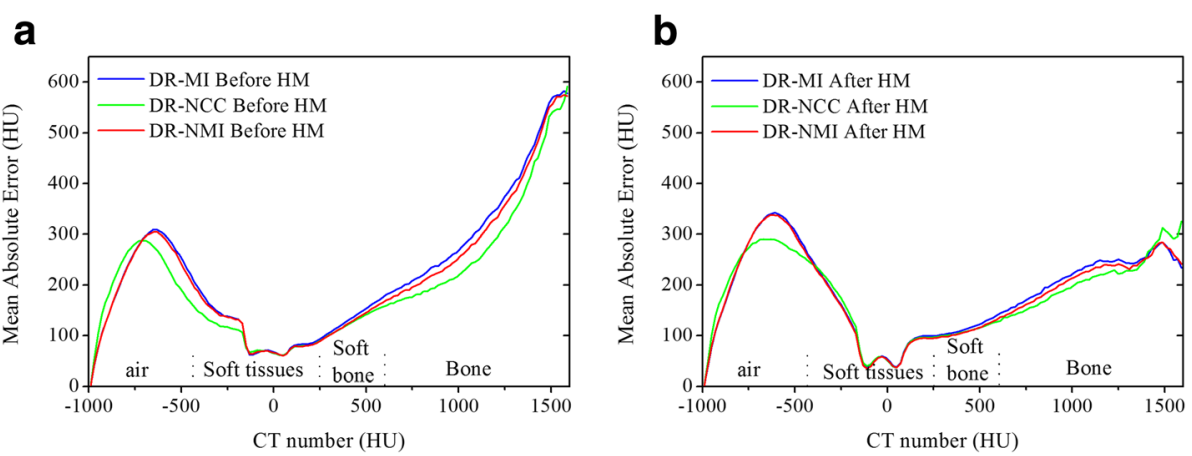

Fig. 5 MAE values of CBCT images for each algorithm before HM (a) and after HM (b) 
Table 3 Comparison of the mean pixel values in fat and muscle between the $\mathrm{PCT}, \mathrm{CBCT}$ and corrected $\mathrm{CBCT}$ images with the three different DR algorithms

\begin{tabular}{|c|c|c|c|c|c|}
\hline \multicolumn{6}{|c|}{ Mean pixel values [HU] } \\
\hline \multirow{2}{*}{$\begin{array}{l}\text { Patient's } \\
\text { number }\end{array}$} & \multicolumn{5}{|l|}{ Fat } \\
\hline & $\mathrm{pCT}$ & $\mathrm{CBCT}$ & $C B C T_{D R-N C C}$ & $C B C T_{D R-M I}$ & $\mathrm{CBCT}_{\mathrm{DR}-\mathrm{NMI}}$ \\
\hline 1 & -108.16 & -144.37 & -90.73 & -92.50 & -92.06 \\
\hline 2 & -87.68 & -163.92 & -84.98 & -88.25 & -85.77 \\
\hline 3 & -104.83 & -139.75 & -90.03 & -91.48 & -91.16 \\
\hline 4 & -103.80 & -151.60 & -104.64 & -105.23 & -105.19 \\
\hline 5 & -103.43 & -170.34 & -102.28 & -103.16 & -102.89 \\
\hline 6 & -109.43 & -130.55 & -98.50 & -99.30 & -99.09 \\
\hline 7 & -97.70 & -148.56 & -97.08 & -98.47 & -97.97 \\
\hline 8 & -108.62 & -213.92 & -108.76 & -108.53 & -108.04 \\
\hline 9 & -110.23 & -160.35 & -105.87 & -106.76 & -106.82 \\
\hline 10 & -99.50 & -123.33 & -100.24 & -101.55 & -101.40 \\
\hline \multirow[t]{2}{*}{ RMSD } & & 56.83 & 8.19 & 7.38 & 7.57 \\
\hline & Muscle & & & & \\
\hline 1 & 49.45 & -1.37 & 31.76 & 30.62 & 30.68 \\
\hline 2 & 51.61 & 25.39 & 42.09 & 41.12 & 41.68 \\
\hline 3 & 47.59 & 59.00 & 53.25 & 54.53 & 53.25 \\
\hline 4 & 42.17 & 45.81 & 50.65 & 51.61 & 50.93 \\
\hline 5 & 48.87 & 11.70 & 47.66 & 47.66 & 47.65 \\
\hline 6 & 49.68 & 40.03 & 50.09 & 50.78 & 50.25 \\
\hline 7 & 49.73 & 28.39 & 45.44 & 46.12 & 46.01 \\
\hline 8 & 46.46 & 13.39 & 36.65 & 39.04 & 39.34 \\
\hline 9 & 48.85 & 56.30 & 52.18 & 51.95 & 51.86 \\
\hline 10 & 49.44 & 51.98 & 45.97 & 47.25 & 46.40 \\
\hline RMSD & & 25.49 & 7.72 & 8.28 & 8.02 \\
\hline
\end{tabular}

was clearly visualised. In addition, the sensitivity of HM process to the quality of registration has been proved. It has been found that the better the alignment the more significant is the $\mathrm{HM}$ contribution to correct the $\mathrm{HU}$ distribution in $\mathrm{CBCT}$ images. For that reason, the best compromise for this correction method seems to be the use of DR with NCC similarity metric for which the MAE values after correction were found to be the lowest as indicated in Fig. 5. Also, this choice can be justified by Table 2 where reduced HU errors in corrected CBCT were obtained for the DR-NCC algorithm.

Despite the influence of DR accuracy on the HM process, the use of DR-NCC before HM yielded acceptable HU errors values compared to other studies investigating pCT-based approaches and direct approaches without recourse to pCT [29, 30]. In [29], Kida et al. applied a deep convolutional neural network (DCNN) method to improve the quality of $\mathrm{CBCT}$ images acquired for 20 prostate cancer patients. They reported that the RMSD of the mean pixel values for corrected
CBCT images was about 11 and $14 \mathrm{HU}$ in fat and muscle, respectively, while in our study the same evaluation showed that the RMSD was about $8 \mathrm{HU}$. This suggests that our proposed workflow had successfully improved the spatial uniformity in the CBCT images. Besides, Poludniowski et al. [30] studied for 12 patients (6 brain, 3 prostate and 3 bladder cancer patients) four correction methods based on "scatter calibration" and "scatter measurement" using CBCT images acquired by other linear accelerator (Elekta Linac). They reported that for prostate cancer the average $\mathrm{HU}$ error for each method, called also Root Mean Squared Difference, were about 95.5, 91.5, 73.1 and $67.7 \mathrm{HU}$. Whereas, in our study the HM considered as pCT-based approach resulted in average $\mathrm{HU}$ error of about $55.95 \pm 10.43 \mathrm{HU}$ when using DR-NCC indicating that although our CBCT images differs from theirs; our results are better than their findings.

To improve the correctness of the proposed workflow, the minimization of its limitations as the existence of non-comparable regions in the $\mathrm{pCT}$ and $\mathrm{CBCT}$ images, e.g. regions of gas in the rectum, is a priority. Thus, further investigations taking into account the correction of these regions before performing DR and HM are required. In addition, dosimetric evaluation is needed to validate the efficiency of using corrected $\mathrm{CBCT}$ for dose calculation in the context of adaptive radiation therapy. Also, we are looking forward to applying this workflow on large number of patients and translate it to other body regions.

\section{Conclusion}

In this study, the impact of using different DR algorithms on the HM process to correct CBCT images was evaluated. The results showed that the quality of correction is strongly dependent to the accuracy of DR process and revealed that performing HM after DR with the NCC similarity metric contributed significantly to reduce the uncertainties in CBCT images. On the basis of this study, a combination of the present workflow with automatic segmentation algorithms could be a promising way towards online adaptive radiation therapy.

\section{Abbreviations}

ART: Adaptive Radiation Therapy; ASGD: Adaptive Stochastic Gradient Descent; CBCT: Cone Beam Computed Tomography; DCNN: Deep Convolutional Neural Network; DR: Deformable Registration; DRMI: Deformable Registration based on Mutual Information similarity metric; DR-NCC: Deformable Registration based on Normalized Correlation Coefficient similarity metric; DR-NMI: Deformable Registration based on Normalized Mutual Information similarity metric; HM: Histogram Matching; HU: Hounsfield Unit; LUT: Look Up Table; MAE: Mean Absolute Error; MLT: Multilevel Threshold; OBI: On-Board Imager; pCT: Planning Computed Tomography; RMSD: Root Mean Squared Difference; ROls: Regions of Interest RR-MI: Rigid Registration based on Mutual Information similarity metric; $V_{\text {err: }}$ Average $\mathrm{HU}$ error over the entire volume 


\section{Acknowledgements}

Not applicable.

\section{Funding}

Not applicable.

\section{Availability of data and materials}

The datasets used and analyzed during the current study are available from the corresponding author on reasonable request.

\section{Authors' contribution}

HS K designed and executed the experiment process, analyzed and interpreted the results and was major contributor in writing the manuscript. HA supervised the whole study. Both authors read and approved the final manuscript.

\section{Ethics approval and consent to participate}

Not applicable.

\section{Consent for publication}

Not applicable.

\section{Competing of interests}

The authors declare that they have no competing interests.

\section{Publisher's Note}

Springer Nature remains neutral with regard to jurisdictional claims in published maps and institutional affiliations.

Received: 24 May 2018 Accepted: 22 October 2018

Published online: 07 November 2018

\section{References}

1. Letourneau D, Martinez AA, Lockman D, et al. Assessment of residual error for online cone-beam CT-guided treatment of prostate cancer patients. Int J Radiat Oncol Biol Phys. 2005;62:1239-46.

2. Oldham M, et al. Cone-beam CT guided radiation therapy: a model for online application. Radiother Oncol. 2005;75:271.e1-271.e8.

3. Gukenberger M, Meyer J, Vordermark D, Baier K, et al. Magnitude and clinical relevence of translational and rotational patient setup errors: a conebeam CT study. Int J Radiat Oncol Biol Phy. 2006;65:934-42.

4. Streink MF, Bezak E. Technological approaches to in-room CBCT imaging. Phys Eng Sci Med Australas. 2008;31:167-79.

5. Jaffray DA, Siewerdsen JH, Wong JW, Martinez AA. Flat-panel cone-beam computed-tomography for image-guided radiation therapy. Int J Radiat Oncol Biol Phys. 2002;53:1337-49.

6. Lafond C, Simon A, Henry O, Prichon N. Radiothérapie adaptative en routine? Etat de l'art: point de vue du physicien médical. Cancer Radiothérapie. 2015; In press.

7. Kim S, Yoo S, Yin FF. Kilovoltage cone-beam CT: comparative dose and image quality evaluation in partial and full-angle scan protocols. Med Phys. 2010;37:3648-459

8. Stock S, Pasler M, Birkfellner W. Image quality and stability of image-guided radiotherapy (IGRT) devices: a comparatives study. Radiother Oncol. 2009;93:1-7.

9. Zijtveld MV, Dirkx M, Heijmen B. Correction of cone beam CT values using a planning CT for derivation of the dose of the day. Radiother Oncol. 2007;85: 195-200.

10. Yang Y, Schreibmann E, Li T, Wang C, Xing L. Evaluation of on-board kv cone beam CT (CBCT)-based dose calculation. Phys Med Biol. 2007;52:685-705.

11. Boggula R, Lorenz F, Abo-Madyan Y, Lohr F, Wolff D, Boda-Heggemann J, et al. A new strategy for online adaptive prostate radiotherapy on cone-beam CT. Med Phys. 2009;19:264-76.

12. Fotina I, Hopfgartner J, Stock M, Steininger T, Ltegendorf-Cancig C, George D. Feasibility of CBCT-based dose calculation: comparative analysis of $\mathrm{HU}$ adjustment techniques. Radiother Oncol. 2012;104:249-56.

13. Onozato Y, Kadoy N, Fujita Y, Arai K, Dobashi S, Tekeda K, et al. Evaluation of on board kv cone-beam computed tomography-based dose calculation with deformable image registration using hounsfeild unit modification. Int J Radiot Oncol Biol Phys. 2014;89:416-23.

14. Almatani T, Hugtenburg RP, Lewis RD, Barley SE, Edwards MA. Automated algorithm for CBCT- based dose calculations of prostate radiotherpay with bilateral hip protheses. Br J Radiol. 2016;89:20160443.
15. Amit G, Purdie TG. Automated planning of breast radiotherapy using cone beam CT imaging. Med Phys. 2015:42:770-9.

16. Yoo S, Yin FF. Dosimetric feasibility of cone-beam CT-based treatment planning compared to CT-based treatment planning. Int J Radiat Oncol Biol Phys. 2006;66(5):1553-61.

17. Letourneau $\mathrm{D}$, Wong $\mathrm{R}$, Moseley $\mathrm{D}$, et al. Online planning and delivery technique for radiotherapy of spinal metastases using cone-beam CT: image-quality and system performance. Int J Radiat Oncol Biol Phys. 2007; 67(4):1229-37.

18. Ritcher A, Hu Q, Steglich D, et al. Investigation of the usability of cone beam CT data sets for dose calculation. Radiat Oncol. 2008:3:42.

19. Jarry G, Graham SA, Moseley DJ, et al. Characterization of scattered radiation in kV CBCT images using Monte Carlo simulations. Med Phys. 2006;33(11): $4320-9$

20. Zhu L, Xie $Y$, Wang J, et al. Scatter correction for cone-beam $C T$ in radiation therapy. Med Phys. 2009;36(6):2258-68.

21. Sun M, Star-lack JM. Improved scatter correction using adaptive scatter kernel superposition. Phys Med Biol. 2010;55(22):6695-720.

22. Poludniowski G, Evans PM, Hansen VN, et al. An efficient Monte Carlo-based algorithm for scatter correction in keV cone-beam CT. Phys Med Biol. 2009; 56(12):3847-64.

23. Poludniowski G, Evans PM, Kavanagh A, et al. Removal and effects of scatter-glare in cone-beam CT with an amorphous-silicon flat-panal detector. Phys Med Biol. 2011;56(6):1837-51.

24. Schindelin J, Arganda-Carreras I, Frise E, et al. Fiji: an open-source platform for biological-image analysis. Nat Methods. 2012;9(7):676-82.

25. Fedorov A, Beichel R, Kalpathy-Cramer J, Finet J, et al. 3D slicer as an image computing platform for the quantitative imaging network. Magn Reas Imaging. 2012;30:1323-41.

26. Klein S, Staring M, Murphy K, Viergever MA, Pluim JPW. Elastix: a toolbox for intensity based medical image registration. IEEE Trans Med Imaging. 2010; 29(1):196-205.

27. Thing RS, Bernchou U, Mainegra-Hing E, et al. Hounsfield unit recovery in clinical coen beam $\mathrm{CT}$ images of the thorax acquired for image guided radiation therapy. Phys Med Biol. 2016;61:5781-802.

28. Boydev C, et al. Zero echo time MRI-only treatment planning for radiation therapy of brain tumors after resection. Phys Med. 2017; In press.

29. Kida S, Nakano M, et al. Cone beam computed tomography image improvement using a deep convolutional neural network. Cureus. 2018; 10(4):e2548.

30. Poludniowski G, Evans PM, Webb S. Cone beam computed tomography number errors and consequences for radiotherapy planning: an investigation of correction methods. Int J Radiat Oncol Biol Phys. 2012;84(1): e109-14.

Ready to submit your research? Choose BMC and benefit from:

- fast, convenient online submission

- thorough peer review by experienced researchers in your field

- rapid publication on acceptance

- support for research data, including large and complex data types

- gold Open Access which fosters wider collaboration and increased citations

- maximum visibility for your research: over $100 \mathrm{M}$ website views per year

At $\mathrm{BMC}$, research is always in progress.

Learn more biomedcentral.com/submission 